Kragujevac Journal of Mathematics

Volume 42(4) (2018), Pages 505-516.

\title{
NEW FIXED POINT RESULTS IN ORTHOGONAL METRIC SPACES WITH AN APPLICATION
}

\author{
T. SENAPATI ${ }^{1}$, L. K. DEY ${ }^{2}$, B. DAMJANOVIĆ ${ }^{3}$, AND A. CHANDA $^{4}$
}

\begin{abstract}
In this manuscript, owing to the concept of $w$-distance, we prove the much acclaimed Banach's fixed point theorem in orthogonal metric spaces. Further, our paper includes a couple of illustrative examples which exhibit the purpose for such inquests. In fact, the obtained results extend and improve certain comparable results of existing literature. Eventually, our findings allow us to obtain the existence and uniqueness of solutions of nonlinear fractional differential equations associated with the Caputo fractional derivative.
\end{abstract}

\section{Introduction}

On account of the fact that the metric fixed point theory plays a crucial role in solving many problems in different branches of science, many authors went into the possibility of altering the concepts of metric and metric spaces (see for examples, $[3,7,8])$. Lately, Gordji et al. [4] coined an interesting notion of the orthogonal sets and then, orthogonal metric spaces. Subsequently, they gave an extension of Banach fixed point theorem in this newly defined structure and also, applied their obtained results to prove the existence of a solution of an ordinary differential equation. Furthermore, in [1], the authors improved the results of [4] and also proved a fixed point result concerning $F$-contraction in this setting. Throughout this article, the notations $\mathbb{Z}, \mathbb{N}, \mathbb{R}, \mathbb{R}^{+}$have their usual meanings.

To start with, we recall the definition of an orthogonal set given in [4].

Definition 1.1. Let $X$ be a non-empty set and $\perp$ be a binary relation defined on $X \times X$. Then $(X, \perp)$ is said to be an orthogonal set $\left(O\right.$-set) if there exists $x_{0} \in X$

Key words and phrases. Orthogonal metric space, $w$-distance, fixed point, nonlinear fractional differential equation.

2010 Mathematics Subject Classification. 47H10

Received: April 08, 2017.

Accepted: May 17, 2017. 
such that

$$
(\forall y \in X) x_{0} \perp y \vee(\forall y \in X) y \perp x_{0} .
$$

The element $x_{0}$ is called an orthogonal element. An orthogonal set may have more than one orthogonal element.

Definition 1.2. Let $(X, \perp)$ be an orthogonal set ( $O$-set). Any two elements $x, y \in X$ are said to be orthogonally related if $x \perp y$.

The following is a non-trivial example of an orthogonal set.

Example 1.1. Let $X$ be a non-empty set and we consider the power set $\mathcal{P}(X)$. We define ' $\perp$ ' on $\mathcal{P}(X)$ as $A \perp B$ if $A \cap B=\phi$. Then $(\mathcal{P}(X), \perp)$ is an orthogonal set, as for all $A \in \mathcal{P}(X), \phi \cap A=\phi$. Similarly, one can define ' $\perp$ ' on $\mathcal{P}(X)$ as $A \perp B$ if $A \cup B=X$. Then $(\mathcal{P}(X), \perp)$ is also an orthogonal set.

Let $(X, \perp)$ be an orthogonal set and $d$ be a usual metric on $X$. Then $(X, \perp, d)$ is called an orthogonal metric space ( $O$-metric space).

In [4], the authors defined the concept of orthogonal sequence, completeness and orthogonal continuity which are discussed below.

Definition 1.3. [4] Let $(X, \perp)$ be an orthogonal set $\left(O\right.$-set). Then a sequence $\left(x_{n}\right)$ is said to be an orthogonal sequence ( $O$-sequence) if

$$
(\forall n \in \mathbb{N}) x_{n} \perp x_{n+1} \vee(\forall n \in \mathbb{N}) x_{n+1} \perp x_{n} .
$$

Similarly, a Cauchy sequence $\left(x_{n}\right)$ is said to be a Cauchy $O$-sequence if

$$
(\forall n \in \mathbb{N}) x_{n} \perp x_{n+1} \vee(\forall n \in \mathbb{N}) x_{n+1} \perp x_{n} .
$$

Definition 1.4. [4] An orthogonal metric space $(X, \perp, d)$ is said to be a complete $O$-metric space $(O$-complete) if every Cauchy $O$-sequence converges in $X$.

Definition 1.5. [4] Let $(X, \perp, d)$ be an orthogonal metric space. A function $f: X \rightarrow$ $X$ is said to be orthogonally continuous (O-continuous) at $x$ if for each $O$-sequence $\left(x_{n}\right)$ converging to $x$ implies $f\left(x_{n}\right) \rightarrow f(x)$ as $n \rightarrow \infty$.

The authors of [4] showed that the $O$-continuity is weaker than the standard continuity in standard metric spaces. Also, they raised the following open problem.

Open Problem 1.1. Let $X$ be an inner product space with the inner product $\langle\cdot, \cdot\rangle$. We define $x \perp y$ if $\langle x, y\rangle=0$. Let $f: X \rightarrow X$ be $O$-continuous on $X$. Is $f$ continuous?

The authors did not give any answer to this problem even in the standard inner product space $\mathbb{R}^{n}$. It is quite interesting to find a solution of such type of fundamental questions in the standard inner product space $\mathbb{R}^{n}$ equipped with above mentioned orthogonality relation. We give an affirmative answer to this question in Section 2.

In 1996, Kada et al. [5] introduced the idea of $w$-distance in metric spaces and established several well-known results using this concept. They defined the $w$-distance as follows. 
Definition 1.6. [5] Let $(X, d)$ be a metric space. A function $p: X \times X \rightarrow[0, \infty)$ is said to be a $w$-distance if

$(w 1) p(x, z) \leq p(x, y)+p(y, z)$ for any $x, y, z \in X$

$(w 2)$ for any $x \in X, p(x, \cdot): X \rightarrow[0, \infty)$ is lower semi-continuous;

$(w 3)$ for any $\epsilon>0$, there exists $\delta>0$ such that $p(z, x) \leq \delta$ and $p(z, y) \leq \delta$ imply $d(x, y) \leq \epsilon$.

Remark 1.1. Note that a $w$-distance function $p$ may not be symmetric and also it is possible that $p(x, x) \neq 0$ for some $x$, that is, $p(x, y)=0$ does not imply $x=y$.

The readers are refereed to [5] for some examples and crucial properties of $w$ distances.

Before moving on further, we define orthogonal lower semi-continuity (briefly, $O$ LSC) and then we show that $O$-LSC is weaker than $O$-continuity as well as lower semi-continuity.

Definition 1.7. Let $(X, \perp, d)$ be an $O$-metric space. A function $f: X \rightarrow[0, \infty]$ is said to be $O$-LSC at $x$ if for every $O$-sequence $\left(x_{n}\right)$ converging to $x$, we have

$$
\liminf _{n \rightarrow \infty} f\left(x_{n}\right) \geq f(x) .
$$

The following example shows that $O$-LSC is weaker than $O$-continuity.

Example 1.2. Let $X=\mathbb{R}$. Define $x \perp y$ such that either $x, y \in\left(n-\frac{1}{5}, n+\frac{1}{5}\right)$ for some $n \in \mathbb{Z}$ or $x=0$. Then, clearly $(X, \perp)$ is an $O$-set. We consider the usual metric $d$ on $X$. Then $(X, \perp, d)$ is an $O$-metric space. Let $f: X \rightarrow X$ be defined as

$$
f(x)=\lceil x\rceil .
$$

We claim that this function is not $O$-continuous but it is $O$-lower semi-continuous. Let $\left(x_{n}\right)$ be a non-constant $O$-sequence converging to an integer $l$. Then there exists some $n_{0} \in \mathbb{N}$ such that $x_{n} \in\left(l-\frac{1}{5}, l+\frac{1}{5}\right)$ for all $n>n_{0}$. Then if $x_{n} \rightarrow l$ from left, then $\lim _{n \rightarrow \infty} f\left(x_{n}\right)=l$ and if $x_{n} \rightarrow l$ from right, then $\lim _{n \rightarrow \infty} f\left(x_{n}\right)=l+1$. Therefore, we have

$$
\liminf _{n \rightarrow \infty} f\left(x_{n}\right) \geq f(l) .
$$

This shows that $f$ is an $O$-lower semi-continuous function but it is not $O$-continuous.

The next illustrative example shows that $O$-LSC is in fact weaker than lower semicontinuity.

Example 1.3. Let $X=[0, \infty)$ and we define $x \perp y$ if $x y \leq x$ or $x y \leq y$. Then, for all $x \in X, 0 \perp x$, so $(X, \perp)$ is an $O$-set. We consider the usual metric $d$ on $X$. Then $(X, \perp, d)$ is an $O$-metric space. Let $f: X \rightarrow X$ be defined as

$$
f(x)= \begin{cases}2, & x \in[0,1) \\ 1, & x=1 \\ \frac{1}{2}, & x>1\end{cases}
$$


We show that this function is neither lower semi-continuous nor $O$-continuous but it is an $O$-lower semi-continuous function. We consider the point $x=1$. Let $\left(x_{n}\right)$ be a non-constant sequence converging to 1 . So we have either $f\left(x_{n}\right)=2$ or $f\left(x_{n}\right)=\frac{1}{2}$ for all $n \in \mathbb{N}$ which shows that

$$
\liminf _{n \rightarrow \infty} f\left(x_{n}\right) \geq f(1)
$$

does not hold always. Hence, it is not a lower semi-continuous function at $x=1$. Similarly, one can check that this is not $O$-continuous. Next, we show that this is an $O$-lower semi-continuous function. Let us consider $\left(x_{n}\right)$ be an $O$-sequence converging to 1 . Then, for all $n \in \mathbb{N}, x_{n} \perp x_{n+1} \Rightarrow x_{n} x_{n+1} \leq x_{n}$ or $x_{n+1}$ implies the following two cases.

(a) $x_{n}=1$ and $f\left(x_{n}\right)=1=f(1)$ for all $n \in \mathbb{N}$.

(b) If $\left(x_{n}\right)$ be a non-constant $O$-sequence, then we must have $x_{n}<1$ and $f\left(x_{n}\right)=2$ for all $n \in \mathbb{N}$. Therefore,

$$
\liminf _{n \rightarrow \infty} f\left(x_{n}\right) \geq f(1) .
$$

This shows that $f$ is an $O$-lower semi-continuous function.

From the above two examples it is clear that $O$-LSC is weaker than $O$-continuity as well as lower semi-continuity.

Remark 1.2. Every lower semi-continuous function is $O$-lower semi-continuous but the converse is not true.

Now, we modify the definition of a $w$-distance (Definition 1.6) and the corresponding Lemma 1 presented in [5] in the context of $O$-metric spaces.

Definition 1.8. Let $(X, \perp, d)$ be an $O$-metric space. A function $p: X \times X \rightarrow[0, \infty)$ is said to be a $w$-distance on $X$ if

$\left(w 1^{\prime}\right) p(x, z) \leq p(x, y)+p(y, z)$ for any $x, y, z \in X$;

$\left(w 2^{\prime}\right)$ for any $x \in X, p(x, \cdot): X \rightarrow[0, \infty)$ is $O$-lower semi-continuous;

$\left(w 3^{\prime}\right)$ for any $\epsilon>0$, there exists $\delta>0$ such that $p(z, x) \leq \delta$ and $p(z, y) \leq \delta$ imply $d(x, y) \leq \epsilon$

To prove our main result, we need the following lemma.

Lemma 1.1. Let $(X, \perp, d)$ be an $O$-metric space and $p: X \times X \rightarrow[0, \infty)$ be a $w$-distance. Suppose $\left(x_{n}\right)$ and $\left(y_{n}\right)$ are two $O$-sequences in $X$ and $x, y, z \in X$. Let $\left(u_{n}\right)$ and $\left(v_{n}\right)$ be sequences of positive real numbers converging to 0 . Then we have the following.

(L1) If $p\left(x_{n}, y\right) \leq u_{n}$ and $p\left(x_{n}, z\right) \leq v_{n}$, then $y=z$. Moreover, if $p(x, y)=0$ and $p(x, z)=0$, then $y=z$.

(L2) If $p\left(x_{n}, y_{n}\right) \leq u_{n}$ and $p\left(x_{n}, z\right) \leq v_{n}$, then $y_{n} \rightarrow z$ as $n \rightarrow \infty$.

(L3) If $p\left(x_{n}, x_{m}\right) \leq u_{n}$ for all $m>n$, then $\left(x_{n}\right)$ is a Cauchy O-sequence in $X$.

(L4) If $p\left(x_{n}, y\right) \leq u_{n}$, then $\left(x_{n}\right)$ is a Cauchy O-sequence in $X$.

Proof. Proof is similar to that of Lemma 1 in [5]. 


\section{MAin Results}

We get into this section by stating the following theorem which yields an affirmative answer to the fundamental question related to the Open Problem 1.1.

Theorem 2.1. Let $(X, \perp,\langle\cdot, \cdot\rangle)$ be an orthogonal inner product space where $X=\mathbb{R}^{n}$, $\langle\cdot, \cdot\rangle$ denotes the standard inner product and $\perp$ is an orthogonality relation on $X$ defined as $x \perp y$ if $\langle x, y\rangle=0$. Then $f: X \rightarrow X$ is $O$-continuous on $X$ if and only if $f$ is continuous.

To prove the above theorem, we need the following lemma in the standard inner product spaces.

Lemma 2.1. [6] Let $X=\mathbb{R}^{n}$ be a standard inner product space and $T: X \rightarrow X$ be a mapping where $T(x)=\left(T_{1}(x), T_{2}(x), \ldots, T_{n}(x)\right)$ for all $x \in X$ and each $T_{i}$ is a mapping from $\mathbb{R}^{n}$ to $\mathbb{R}$ for all $i=1,2, \ldots, n$. Then $T$ is continuous at $a=$ $\left(a_{1}, a_{2}, \ldots, a_{n}\right)$ if and only if $T_{i}$ is continuous at a for each $i=1,2, \ldots, n$.

Now we deliver the proof of the above theorem.

Proof. Given that $(X, \perp,\langle\cdot, \cdot\rangle)$ is an orthogonal inner product space where $X=\mathbb{R}^{n}$, $\langle\cdot, \cdot\rangle$ is the standard inner product and $\perp$ is an orthogonality relation on $X$ defined as $x \perp y$ if $\langle x, y\rangle=0$. Suppose, $\left(x_{m}\right)$ is a Cauchy $O$-sequence converging to $x$ where $x_{m}=\left(x_{1}^{m}, x_{2}^{m}, \ldots, x_{n}^{m}\right)$ and $x=\left(x_{1}, x_{2}, \ldots, x_{n}\right)$. Given that $f: X \rightarrow X$ is an $O$-continuous function at $x$, we show that $f$ is also continuous at $x$.

For any $x, y \in X$, the distance function $d(x, y)$ induced by the inner product is given by

$$
d(x, y)=\sqrt{\left(x_{1}-y_{1}\right)^{2}+\left(x_{2}-y_{2}\right)^{2}+\cdots+\left(x_{n}-y_{n}\right)^{2}} .
$$

Since $f$ is $O$-continuous at $x$, for any $O$-sequence $\left(x_{m}\right)$ converging to $x$, we have,

$$
\begin{aligned}
& \lim _{m \rightarrow \infty} d\left(f\left(x_{m}\right), f(x)\right)=0, \\
\Rightarrow & \lim _{m \rightarrow \infty} \sqrt{\left(f_{1}\left(x_{m}\right)-f_{1}(x)\right)^{2}+\left(f_{2}\left(x_{m}\right)-f_{2}(x)\right)^{2}+\cdots+\left(f_{n}\left(x_{m}\right)-f_{n}(x)\right)^{2}}=0, \\
\Rightarrow & \lim _{m \rightarrow \infty}\left(f_{j}\left(x_{m}\right)-f_{j}(x)\right)^{2}=0, \text { for each } j, \\
\Rightarrow & f_{j}\left(x_{m}\right) \rightarrow f_{j}(x), \text { for each } j, \text { as } m \rightarrow \infty, \\
\Rightarrow & f_{j} \text { is continuous at } x, \text { for each } j=1,2, \ldots, n, \\
\Rightarrow & f \text { is continuous at } x \quad \text { (by Lemma } 2.1) .
\end{aligned}
$$

Conversely, let $f$ be any continuous function in $X$. Then it is easy to prove that it is $O$-continuous in $X$. Hence, the proof is completed.

Next, we recall the definition of an orthogonally contraction $(\perp$-contraction) before presenting the definition of an orthogonally $p$-contraction $\left(\perp_{p}\right.$-contraction). 
Definition 2.1. [4] Let $(X, \perp, d)$ be an $O$-metric space. A mapping $T: X \rightarrow X$ is said to be an orthogonally contraction ( $\perp$-contraction) if there exists a $k \in[0,1)$ such that

$$
d(T x, T y) \leq k d(x, y)
$$

for any two orthogonally related elements $x, y \in X$.

Definition 2.2. Let $(X, \perp, d)$ be an $O$-metric space and $p: X \times X \rightarrow[0, \infty)$ be a $w$-distance. A mapping $T: X \rightarrow X$ is said to be an orthogonally $p$-contraction $\left(\perp_{p}\right.$-contraction) if there exists a $k \in[0,1)$ such that

$$
p(T x, T y) \leq k p(x, y),
$$

for all $x, y \in X$ with $x \perp y$.

Remark 2.1. Every $\perp$-contraction is an $\perp_{p}$-contraction for $p(x, y)=d(x, y)$, but every $\perp_{p}$-contraction need not to be an $\perp$-contraction.

The following example illustrates this.

Example 2.1. Let us consider the $O$-metric space $(X, \perp, d)$, where $X=[0,2], d$ is the usual metric on $X$ and $x \perp y$ if $x y \leq x$ or $y$. We define a $w$-distance $p: X \times X \rightarrow X$ by $p(x, y)=y$. Let us define a function $T: X \rightarrow X$ by

$$
T(x)= \begin{cases}\frac{x}{3}, & 0 \leq x \leq \frac{2}{3} \\ 1-x, & \frac{2}{3}<x \leq 1 \\ x-\frac{1}{2}, & x>1\end{cases}
$$

Now if $x \perp y$, then $x y \leq x$ or $x y \leq y$. Let us consider $x y \leq x$. So we have the following cases.

Case 1. Let $x=0$. Then for any $y \in[0,2], x \perp y$. So we get the following.

(i) For $0 \leq y \leq \frac{2}{3}$, then $T x=0$ and $T y=\frac{y}{3}$. So, $p(T x, T y)=T y=\frac{y}{3}$ and $p(T x, T y)=\frac{y}{3} \leq \frac{1}{3} p(x, y)$.

(ii) If $\frac{2}{3}<y \leq 1$, then $T y \in\left[0, \frac{1}{3}\right)$ and $p(T x, T y)=1-y<y=p(x, y)$. In particular, $p(T x, T y) \leq k p(x, y)$, where $k \in\left[\frac{1}{2}, 1\right)$.

(iii) For $y>1$, we have $p(T x, T y)=y-\frac{1}{2} \leq k y=k p(x, y)$, where $k \in\left[\frac{3}{4}, 1\right)$.

Case 2. For all $y \in[0,2]$ and $x=0$, we have $p(T y, T x)=0=k p(x, y)$ for all $k \in[0,1)$.

Case 3. Let $x \neq 0$. Then $y \leq 1$. So, we have:

(i) for $0 \leq y \leq \frac{2}{3}, p(T x, T y) \leq \frac{1}{3} p(x, y)$;

(ii) for $\frac{2}{3}<y \leq 1, p(T x, T y) \leq k p(x, y)$, where $k \in\left[\frac{1}{2}, 1\right)$;

(iii) for $y \leq 1$ and $x>1$, we have $p(T y, T x) \leq k p(y, x)$, where $k \in\left[\frac{3}{4}, 1\right)$. 
The above three cases show that $T$ is an $\perp_{p}$-contraction. But one can observe that $T$ is not an $\perp$-contraction. In particular, we choose $x=1$ and $y=\frac{3}{4}$. Then clearly $x \perp y$. So, $d\left(T(1), T\left(\frac{3}{4}\right)\right)=d\left(0, \frac{1}{4}\right)=\frac{1}{4}$ and also, $d\left(1, \frac{3}{4}\right)=\frac{1}{4}$. Thus we can't find any $k \in[0,1)$ for which $d\left(T(1), T\left(\frac{3}{4}\right)\right) \leq k d\left(1, \frac{3}{4}\right)$ holds.

Before bringing off our main theorem, we recollect the orthogonality preserving $(\perp$-preserving) property of a mapping.

Definition 2.3. [4] A self-map $T$ on an $O$-metric space $(X, \perp, d)$ is said to be orthogonality preserving ( $\perp$-preserving) if $x \perp y$ implies $T x \perp T y$ for all $x, y \in X$.

Theorem 2.2. Let $(X, \perp, d)$ be a complete $O$-metric space with a w-distance $p$. If $T$ is an $\perp_{p}$-contractive, $\perp$-preserving and $O$-continuous self-mapping, then

(a) $T$ has a unique fixed point $\tilde{x} \in X$;

(b) the Picard sequence $\left(T^{n} x\right)$ converges to $\tilde{x}$ for every $x \in X$.

Proof. (a) Let $x_{0}$ be an orthogonal element in $X$ such that

$$
(\forall y \in X) x_{0} \perp y \vee(\forall y \in X) y \perp x_{0} .
$$

We define a sequence $\left(x_{n}\right)$ by $x_{n}=T\left(x_{n-1}\right)=T^{n} x_{0}$. By the property of $\perp$-preserving of $T$, one can easily check that $\left(x_{n}\right)$ is an $O$-sequence, i.e.,

$$
(\forall n \in \mathbb{N}) x_{n} \perp x_{n+1} \vee(\forall n \in \mathbb{N}) x_{n+1} \perp x_{n} .
$$

Now, since $T$ is $\perp_{p}$-contractive and for all $n \in \mathbb{N}, x_{n}, x_{n+1}$ are orthogonally related, so we derive

$$
\begin{aligned}
p\left(T x_{n-1}, T x_{n}\right) & \leq k p\left(x_{n-1}, x_{n}\right) \\
\Rightarrow p\left(x_{n}, x_{n+1}\right) & \leq k p\left(x_{n-1}, x_{n}\right) \\
& \leq k^{2} p\left(x_{n-2}, x_{n-1}\right) \\
& \vdots \\
& \leq k^{n} p\left(x_{0}, x_{1}\right) .
\end{aligned}
$$

Using this for all $m>n$, we have,

$$
\begin{aligned}
p\left(x_{n}, x_{m}\right) & \leq p\left(x_{n}, x_{n+1}\right)+p\left(x_{n+1}, x_{n+2}\right)+\cdots+p\left(x_{m-1}, x_{m}\right) \\
& \leq p\left(x_{0},, x_{1}\right)\left[k^{n}+k^{n+1}+\cdots+k^{m-1}\right] \\
& \leq \frac{k^{n}}{1-k} p\left(x_{0}, x_{1}\right) .
\end{aligned}
$$

Let us define $u_{n}=\frac{k^{n}}{1-k} p\left(x_{0}, x_{1}\right)$. Clearly, $u_{n} \rightarrow 0$ as $n \rightarrow \infty$. So by (L3), we must have that $\left(x_{n}\right)$ is a Cauchy $O$-sequence. Since $(X, \perp, d)$ is a complete $O$-metric space, so $x_{n} \rightarrow \tilde{x} \in X$. Now, we show that $\tilde{x}$ is a fixed point of $T$. 
By using $O$-continuity of $T$, we obtain

$$
d(\tilde{x}, T \tilde{x})=\lim _{n \rightarrow \infty} d\left(x_{n+1}, T \tilde{x}\right)=\lim _{n \rightarrow \infty} d\left(T\left(x_{n}\right), T \tilde{x}\right)=d(T \tilde{x}, T \tilde{x})=0 .
$$

This shows that $\tilde{x}$ is a fixed point of $T$.

Now our intention is to show that this $\tilde{x}$ is the unique fixed point of $T$. Let $\tilde{x}$ and $\tilde{y}$ be two fixed points of $T$. Therefore, we have

$$
\left(x_{0} \perp \tilde{x} \wedge x_{0} \perp \tilde{y}\right) \vee\left(\tilde{x} \perp x_{0} \wedge \tilde{y} \perp x_{0}\right) .
$$

Since $T$ is $\perp$-preserving, so for all $n \in \mathbb{N}$, we have

$$
\left(T^{n} x_{0} \perp \tilde{x} \wedge T^{n} x_{0} \perp \tilde{y}\right) \vee\left(\tilde{x} \perp T^{n} x_{0} \wedge \tilde{y} \perp T^{n} x_{0}\right) .
$$

Using $\perp_{p^{-}}$-contractivity condition of $T$, we get

$$
p\left(x_{n}, \tilde{x}\right)=p\left(T^{n} x_{0}, T^{n} \tilde{x}\right) \leq k^{n} p\left(x_{0}, \tilde{x}\right)
$$

and

$$
p\left(x_{n}, \tilde{y}\right)=p\left(T^{n} x_{0}, T^{n} \tilde{y}\right) \leq k^{n} p\left(x_{0}, \tilde{y}\right) .
$$

Let us consider, $u_{n}=k^{n+1} p\left(x_{0}, \tilde{x}\right)$ and $v_{n}=k^{n+1} p\left(x_{0}, \tilde{y}\right)$. Clearly, $\left(u_{n}\right)$ and $\left(v_{n}\right)$ are two sequences of real numbers converging to 0 . Hence by (L1) of Lemma 2.1, we obtain $\tilde{x}=\tilde{y}$, i.e., $T$ has a unique fixed point.

(b) We show that for any $y \in X$, the Picard sequence $\left(T^{n} y\right)$ converges to $\tilde{x}$. Since $(X, \perp)$ is an orthogonal set, so $\left(x_{0} \perp y\right) \vee\left(y \perp x_{0}\right)$, which implies that

$$
\begin{gathered}
(\forall n \in \mathbb{N}) T^{n} x_{0} \perp T^{n} y \vee(\forall n \in \mathbb{N}) T^{n} y \perp T^{n} x_{0} . \\
p\left(T^{n} x_{0}, T^{n} y\right) \leq k^{n} p\left(x_{0}, y\right)=\alpha_{n} .
\end{gathered}
$$

Again, we already have

$$
p\left(x_{n}, \tilde{x}\right)=p\left(T^{n} x_{0}, T^{n} \tilde{x}\right) \leq k^{n} p\left(x_{0}, \tilde{x}\right)=\beta_{n} .
$$

As $\left(\alpha_{n}\right)$ and $\left(\beta_{n}\right)$ are two sequences of real numbers converging to 0, so (L2) of Lemma 2.1 implies that $T^{n} y \rightarrow \tilde{x}$.

Hence, the proof is complete.

The following theorem shows that $O$-continuity property of $T$ is not necessary to prove the existence of a fixed point of $T$. By adding the following hypothesis with Theorem 2.2, one can also get the same result.

Theorem 2.3. Let $x_{0}$ be an orthogonal element such that the Picard sequence $\left(T^{n} x_{0}\right)$ converges to $\tilde{x}$. If

$$
(\forall n \in \mathbb{N}) T^{n} x_{0} \perp \tilde{x} \vee(\forall n \in \mathbb{N}) \tilde{x} \perp T^{n} x_{0},
$$

then $\tilde{x}$ is the unique fixed point of $T$. 
Proof. Suppose the Picard sequence $\left(x_{n}\right) \rightarrow \tilde{x}$ as $n \rightarrow \infty$ and $x_{n} \perp \tilde{x}$, where $x_{n}=$ $T^{n} x_{0}$. Using the $O$-lower semi-continuity property of $p$, we get

$$
p\left(x_{n+1}, \tilde{x}\right) \leq \liminf _{n \rightarrow \infty} p\left(x_{n+1}, x_{n+m}\right) \leq \liminf _{n \rightarrow \infty} \frac{k^{n-1}}{1-k} p\left(x_{0}, x_{1}\right)=0 .
$$

Since $T$ is $\perp$-preserving and $x_{n} \perp \tilde{x}$, so

$$
p\left(T x_{n}, T \tilde{x}\right) \leq k p\left(x_{n}, \tilde{x}\right) \leq k \liminf _{n \rightarrow \infty} p\left(x_{n}, x_{n+m}\right) \leq \liminf _{n \rightarrow \infty} \frac{k^{n+1}}{1-k} p\left(x_{0}, x_{1}\right)=0 .
$$

By (L1) of Lemma 2.1, we must have $T \tilde{x}=\tilde{x}$, i.e., $\tilde{x}$ is a fixed point of $T$. Uniqueness of the fixed point can be proved as in the previous theorem.

Next, we illustrate the validity of our results by the following example.

Example 2.2. We recall the Example 2.1. It is clear that $(X, \perp, d)$ is a complete $O$-metric space, $T$ is an $\perp_{p}$-contraction and $\perp$-preserving. Again, we have already observed that $T$ is not an $\perp$-contraction. So we can't use Theorem 3.11 of [4] to find a fixed point of $T$. But, here we can apply our results to prove the existence of a fixed point of $T$. It is obvious that every $x \in[0,1]$ is an orthogonal element and the Picard sequence $\left(T^{n} x\right)$ converges to 0 for every $x \in[0,1]$. Note that the mapping $T$ is not $O$-continuous on $X$. Let us consider a point $x=\frac{2}{3}$ and we choose a sequence $\left(x_{n}\right)$ from $\left(\frac{2}{3}, 1\right]$ which converges to $\frac{2}{3}$. Clearly, for all $n, x_{n} \perp x_{n+1}$, i.e., $\left(x_{n}\right)$ is an $O$-sequence converging to $\frac{2}{3}$ but $\lim _{n \rightarrow \infty} T\left(x_{n}\right) \neq T\left(\frac{2}{3}\right)$. For every $n \in \mathbb{N}$ and for all $x \in[0,1], 0 \perp T^{n} x$. By the assumptions of Theorem 2.3, $x=0$ is the fixed point of $T$. Also, note that for any $x \in[0,2]$, the Picard sequence converges to 0 and $p(0,0)=0$.

Remark 2.2. If we set $p(x, y)=d(x, y)$, then Theorem 3.11 of [4] can be obtained from our Theorem 2.2.

\section{Application}

In this section we employ our main result in nonlinear fractional differential equations. Here, we find a solution for the following nonlinear fractional differential equation (see [2]) given by

$$
{ }^{C} D^{\beta} x(t)=f(t, x(t)) \quad(0<t<1,1<\beta \leq 2),
$$

with boundary conditions

$$
x(0)=0, x^{\prime}(0)=I x(t) \quad(0<t<1),
$$

where ${ }^{C} D^{\beta}$ stands for the Caputo fractional derivative of order $\beta$ which is defined as

$$
{ }^{C} D^{\beta} f(t)=\frac{1}{\Gamma(n-\beta)} \int_{0}^{t}(t-s)^{n-\beta-1} f^{n}(s) d s \quad(n-1<\beta<n, n=[\beta]+1),
$$


and $f:[0,1] \times \mathbb{R} \rightarrow \mathbb{R}^{+}$is a continuous function. We consider $X=C([0,1], \mathbb{R})$, the set of all continuous functions from $[0,1]$ into $\mathbb{R}$ with supremum norm $\|x\|_{\infty}=\sup _{t \in[0,1]}|x(t)|$. So, $\left(X,\|.\|_{\infty}\right)$ is a Banach space.

The Riemann-Liouville fractional integral of order $\beta$ (for detail, see [9]) is given by

$$
I^{\beta} f(t)=\frac{1}{\Gamma(\beta)} \int_{0}^{t}(t-s)^{\beta-1} f(s) d s \quad(\beta>0) .
$$

At first, we present an appropriate form of a nonlinear fractional differential equation and then investigate the existence of a solution of the given problem through fixed point theorem. So, we consider the following fractional differential equation

$$
{ }^{C} D^{\beta} x(t)=f(t, x(t)) \quad(0<t<1,1<\beta \leq 2),
$$

with the integral boundary conditions

$$
x(0)=0, x^{\prime}(0)=I x(t) \quad(0<t<1),
$$

where

(a) $f:[0,1] \times \mathbb{R} \rightarrow \mathbb{R}^{+}$is a continuous function,

(b) $x(t):[0,1] \rightarrow \mathbb{R}$ is continuous

satisfying the following conditions

$$
|f(t, x)-f(t, y)| \leq L|x-y|,
$$

for all $t \in[0,1]$ and for all $x, y \in X$ such that $x(t) y(t) \geq 0, L$ is a constant with $L \lambda<1$ where

$$
\lambda=\frac{1}{\Gamma(\beta+1)}+\frac{2 k^{\beta+1} \Gamma(\beta)}{\left(2-k^{2}\right) \Gamma(\beta+2)} .
$$

Then the differential equation (3.1) has a unique solution.

Proof. We consider the following orthogonality relation on $X$

$$
x \perp y \text { if } x(t) y(t) \geq 0,
$$

for all $t \in[0,1]$. Clearly, $(X, \perp)$ is an orthogonal set since for every $x \in X$ there exists $y(t)=0$, for all $t \in[0,1]$ such that $x(t) y(t)=0$. We consider $d(x, y)=$ $\sup _{t \in[0,1]}\|x(t)-y(t)\|$ for all $x, y \in X$. So, $(X, \perp, d)$ is a complete orthogonal metric space.

We define a mapping $T: X \rightarrow X$ by

$$
\begin{aligned}
T x(t)= & \frac{1}{\Gamma(\beta)} \int_{0}^{t}(t-s)^{\beta-1} f(s, x(s)) d s \\
& +\frac{2 t}{\left(2-k^{2}\right) \Gamma(\beta)} \int_{0}^{k} \int_{0}^{s}(s-m)^{\beta-1} f(m, x(m)) d m d s,
\end{aligned}
$$

for $t \in[0,1]$. 
A function $x \in X$ is a solution of Equation (3.1) if and only if $x(t)=T x(t)$ for all $t \in[0,1]$. In order to prove the existence of a fixed point of $T$, we show that $T$ is $\perp$-preserving and $\perp_{p}$-contractive.

At first, we show that $T$ is $\perp$-preserving. Let, for all $t \in[0,1], x(t) \perp y(t)$. Now, we have

$$
\begin{aligned}
T x(t)= & \frac{1}{\Gamma(\beta)} \int_{0}^{t}(t-s)^{\beta-1} f(s, x(s)) d s \\
& +\frac{2 t}{\left(2-k^{2}\right) \Gamma(\beta)} \int_{0}^{k}\left(\int_{0}^{s}(s-m)^{\beta-1} f(m, x(m)) d m\right) d s>0,
\end{aligned}
$$

which implies that $T x \perp T y$, i.e., $T$ is $\perp$-preserving.

Next, we show that $T$ is $\perp_{p}$-contractive. For all $t \in[0,1]$ and $x(t) \perp y(t)$, we obtain

$$
\begin{aligned}
|T x-T y|=\mid & \frac{1}{\Gamma(\beta)} \int_{0}^{t}(t-s)^{\beta-1} f(s, x(s)) d s \\
& +\frac{2 t}{\left(2-k^{2}\right) \Gamma(\beta)} \int_{0}^{k}\left(\int_{0}^{s}(s-m)^{\beta-1} f(m, x(m)) d m\right) d s \\
& -\frac{1}{\Gamma(\beta)} \int_{0}^{t}(t-s)^{\beta-1} f(s, y(s)) d s \\
& -\frac{2 t}{\left(2-k^{2}\right) \Gamma(\beta)} \int_{0}^{k}\left(\int_{0}^{s}(s-m)^{\beta-1} f(m, y(m)) d m\right) d s \mid \\
\leq & \frac{1}{\Gamma(\beta)} \int_{0}^{t}(t-s)^{\beta-1}|f(s, x(s))-f(s, y(s))| d s \\
& +\frac{2}{\left(2-k^{2}\right) \Gamma(\beta)} \int_{0}^{k} \int_{0}^{s}(s-m)^{\beta-1}|f(m, x(m))-f(m, y(m))| d m d s \\
\leq & \frac{L\|x-y\|}{\Gamma(\beta)} \int_{0}^{t}(t-s)^{\beta-1} d s+\frac{2 L\|x-y\|}{\left(2-k^{2}\right) \Gamma(\beta)} \int_{0}^{k} \int_{0}^{s}(s-m)^{\beta-1} d m d s \\
\leq & \frac{L\|x-y\|}{\Gamma(\beta+1)}+\frac{2 k^{\beta+1} L\|x-y\| \Gamma(\beta)}{\left(2-k^{2}\right) \Gamma(\beta+2)} \\
\leq & L\|x-y\|\left(\frac{1}{\Gamma(\beta+1)}+\frac{2 k^{\beta+1} \Gamma(\beta)}{\left(2-k^{2}\right) \Gamma(\beta+2)}\right)
\end{aligned}
$$

which implies $\|T x-T y\| \leq L \lambda\|x-y\|$. Now, if we set $p(x, y)=d(x, y)$, then we have

$$
p(T x, T y) \leq L \lambda p(x, y),
$$

which shows that $T$ is $\perp_{p}$-contractive as $L \lambda<1$.

Next, we consider that $\left(x_{n}\right)$ is a Cauchy $O$-sequence converging to $x$. So, we must have $x_{n}(t) x_{n+1}(t) \geq 0$ for all $t \in[0,1]$ and $n \in \mathbb{N}$. This gives us two possibilities: either $x_{n}(t) \geq 0$ or $x_{n}(t) \leq 0$ for all $n \in \mathbb{N}$ and each $t \in[0,1]$. Let us consider the case $x_{n}(t) \geq 0$ for each $t \in[0,1]$ and $n \in \mathbb{N}$. Then, for every $t \in[0,1], x_{n}(t)$ produces a sequence of nonnegative real numbers which converges to $x(t)$. Hence, we must 
get $x(t) \geq 0$ for each $t \in[0,1]$, i.e., $x_{n}(t) \perp x(t)$ for all $n \in \mathbb{N}$ and $t \in[0,1]$. So, by Theorem 2.3, $x(t)$ is the unique fixed point of $T$ which is the required solution of Equation (3.1).

Acknowledgements. We like to thank the learned referee for careful reading and constructive suggestions which undoubtedly improve the first draft. The first and fourth named authors express their sincere thanks to DST-INSPIRE, New Delhi, India for their financial supports under INSPIRE fellowship scheme.

\section{REFERENCES}

[1] H. Baghani, M. E. Gordji and M. Ramezani, Orthogonal sets: The axiom of choice and proof of a fixed point theorem, J. Fixed Point Theory Appl. 18(3) (2016), 465-477.

[2] D. Baleanu, S. Rezapour and H. Mohammadi, Some existence results on nonlinear fractional differential equations, Philos. Trans. A 371(1990) (2013), 1-7.

[3] L. Ćirić, A generalization of Banach's contraction principle, Proc. Amer. Math. Soc. 45 (1974), 267-273.

[4] M. E. Gordji, M. Ramezani, M. De La Sen and Y. J. Cho, On orthogonal sets and Banach fixed point theorem, Fixed Point Theory 18(2) (2017), 569-578.

[5] O. Kada, T. Suzuki and W. Takahashi, Nonconvex minimization theorems and fixed point theorems in complete metric spaces, Math. Japon. 44(2) (1996), 381-391.

[6] E. Kreyszig, Introductory Functional Analysis with Applications, John Wiley \& Sons, New York, 2003.

[7] B. K. Lahiri, P. Das and L. K. Dey, Cantor's theorem in 2-metric spaces and its applications to fixed point problems, Taiwanese J. Math. 15(1) (2011), 337-352.

[8] T. Senapati, L. K. Dey and D. Dolićanin-Đekić, Extension of Ćirić and Wardowski type fixed point theorems in D-generalized metric spaces, Fixed Point Theory Appl. 2016:33 (2016), 1-14.

[9] W. Sudsutad and J. Tariboon, Boundary value problems for fractional differential equations with three-point fractional integral boundary conditions, Adv. Difference Eqn. 2012:93 (2012), 1-10.

\footnotetext{
${ }^{1}$ Department of Mathematics,

National Institute of Technology Durgapur,

DURGAPUR, INDIA

Email address: senapati.tanusri@gmail.com

${ }^{2}$ Department of Mathematics,

National Institute of Technology Durgapur, DURGAPUR, INDIA

Email address: lakshmikdey@yahoo.co.in

${ }^{3}$ Department of Mathematics, State University of Novi Pazar, Novi Pazar, Serbia

Email address: dambo@agrif.bg.ac.rs

${ }^{4}$ Department of Mathematics,

National Institute of Technology Durgapur, DURGAPUR, INDIA

Email address: ankushchanda8@gmail.com
} 